\title{
GALACTIC DYNAMICS AND GALACTIC DISTRIBUTION
}

\author{
M.W. FEAST \\ (Radcliffe Observatory, South Africa)
}

The main papers at this symposium dealing with the galactic dynamics and distribution of the planetary nebulae were those of Perek and Cahn, and I shall not attempt to summarize their work again. Rather, I want to draw attention to what I believe are some of the main problems we have to solve in order to make further progress in this field.

Aside from the problem of deciding whether a particular object is a planetary or not, a far from trivial problem, there seem to be two basic problems to be solved. First, there is the problem of distance determinations, and second, the problem of the heterogeneous nature of the planetary population in the Galaxy.

Taking the second point first, it seems to me that there are good reasons for believing that the planetaries have a wide range of ages, and that these ages span the period of the initial collapse of the Galaxy to a disk. For instance, the presence of a planetary nebula in the globular cluster M15 which is metal deficient by a factor of the order of 100 , indicates that some planetaries belong to the Halo population. Nevertheless, the overall distribution of planetaries, unlike that of globular clusters, shows a marked concentration to the galactic plane, indicating a substantial fraction of disk objects. Furthermore, the velocity dispersion of the planetary nebulae, radial to the galactic centre, increases steadily as one goes from the Sun in towards the galactic centre. This was shown very clearly by Perek using the velocities of Mayall and Minkowski. This result is paralleled in a remarkable way by the Mira variables. Studies of the Miras have the great advantage that although we know that they cover a rather wide range of ages, they may be separated into groups of relatively small age dispersion by grouping according to the period. Thus, in the case of the Miras, we are able to see that the increase in the velocity dispersion towards the galactic centre is simply a reflection of decreasing mean period; that is, of increasing mean age, as the galactic centre is approached. For Miras of a given period (i.e. of a given age) the velocity dispersion seems to be independent of distance from the centre; a result which, incidentally, is in accord with the predictions of classical ellipsoidal theory. Evidently, therefore, the change in the velocity dispersion of the planetaries with distance from the centre, indicates a range in ages for these objects. A further point is the fact that in the solar neighbourhood the asymmetrical drift for the planetaries $(-16 \pm 7 \mathrm{~km} / \mathrm{sec})$ matches that of the younger (that is, the longer-period) Miras.

Evidently, therefore, before much further progress can be made in discussing the 
galactic distribution and dynamics of the planetaries there is an urgent requirement that we find some physical characteristic of the planetaries which is an age index. Very little seems to be known on this point. Some long while ago, H. M. Johnson (Astrophys. J., 120, 1954, 182) suggested that there was a statistical correlation between the distance of the planetaries from the galactic plane and their excitation class $(\mathrm{N} 2 / \mathrm{H} \beta$ ratio). A much more extensive investigation of this and other possible age-dependent properties of the planetaries is needed. Both Dr Westerlund and I mentioned the different frequency distributions of excitation classes in the two Magellanic Clouds, and in the LMC there is some suggestion of a correlation between absolute magnitude and velocity dispersion for the planetaries. These may perhaps be clues to the type of age index for which we are looking. Clearly, wholesale determinations of chemical abundances would be of very great importance in subdividing the planetaries into coeval groups, but we have heard from Aller of the difficulties of this work.

While we can be fairly certain that there are planetary nebulae ranging in age from the oldest Halo objects down to the younger disk objects, we are quite uncertain as to how old the youngest planetaries can be. Westerlund and Miss Webster have suggested that some of the planetaries in the SMC may have come from stars with masses as large as $1.8 M_{\odot}$. This would make them relatively young objects, and of course there is the interesting case of NGC 1514 discussed by Kohoutek, where the planetary nucleus appears to have an early A-type companion. On the other hand, it seems rather unlikely on kinematic grounds that there can be any very high proportion of extremely young planetaries in the Galaxy. This seems to be shown fairly clearly in the Magellanic Clouds also. In both Clouds, although the planetaries seem to have the same overall kinematic flow pattern, as is shown by the very young supergiants and the gas, there is a lack of detailed agreement of positions and velocities for the planetaries and the very young objects.

It must be emphasized that until we solve this problem of dividing the planetaries into homogeneous groups, we can only study the galactic distribution and dynamics of these objects in a rather limited way.

The other major problem in discussing the distribution and kinematics of the planetaries is the problem of distance determination. A great deal of work has been, and must still be, put into the solution of this problem. It does, however, seem necessary to stress one point which appears to have received little attention up till now. We require not only a means of distance modulus estimation which is correct on the average, but also an estimate of the standard error of the distance modulus. Straightforward statistical arguments show that if the standard error of a modulus determination is high, then large corrections to the mean distances may be necessary when the distribution or kinematics of the planetaries are being studied. This is, of course, a classical problem in statistical astronomy; but since one or two people apparently misunderstood me when I raised the point briefly in the general discussion, I should perhaps give a simple example. If we look in a direction in which the space density of 
the planetaries is increasing with distance, and select all the planetaries to which we have assigned the distance $r$, then this group obviously contains some planetaries whose true distance is greater than $r$ and some whose true distance is less. Because of the density gradient there are more stars at greater $r$ so that a group selected in this way contains more underestimated distances than overestimated distances. The resulting systematic error in the mean value of $r$ may be quite large and of considerable importance in dynamical studies, especially for distant objects in the direction of the galactic centre where the density gradient is large. Evidently here again the Magellanic Clouds should prove invaluable both in helping fix a distance scale as well as in giving some indication of the errors of an individual modulus.

To conclude let me mention three observational problems. Firstly, and obviously, the extension of radial velocity work in the Southern Hemisphere based on the many new Southern planetaries discovered by Henize is very desirable. Secondly, it must now be clear to all of us just how important a place in our ideas is occupied by the planetary in M 15. It would be a great reassurance if we could find at least a couple of field planetaries that also have low $\mathrm{O} / \mathrm{H}$ and $\mathrm{Ne} / \mathrm{H}$ ratios. Finally, there has been an indication this week that more planetaries are expected in globular clusters than are actually found. Further searches for such objects are evidently desirable, and it is important that investigators whose searches have proved negative be encouraged to publish full details of their work.

\section{DISCUSSION}

O'Dell: The suggestion by H.M. Johnson that there exist variations in the excitation of planetaries correlated with galactic latitude is not confirmed by photoelectric measurements. The apparent effect seen by Johnson was probably due to uncertainties in the photographic spectrophotometry.

I believe that the expected number of planetaries that should exist in globular clusters is about 5 in the entire Galaxy, which indicates that we are at about the correct level of discovery since only one nebula has been seen.

Minkowski: One should consider not only planetary nebulae in globular clusters, but also planetaries at large distances in the halo. The only known object of this kind is a planetary of about magnitude 15 close to the galactic pole discovered by Haro. A search for more objects of this kind might be of interest.

Feast: While I think that a search for planetaries far from the galactic plane would be very valuable, I would guess from the distribution of the much more frequent Mira variables that one would not perhaps expect to find many such planetaries, so that the search would have to be very extensive. 